\title{
Statistics of resonance states in a weakly open chaotic microwave cavity
}

\author{
C. POLI, O. LEGRAND and F. MORTESSAGNE \\ Laboratoire de Physique de la Matière Condensée (LPMC) \\ CNRS UMR 6622 \& Université de Nice-Sophia Antipolis
}

in collaboration with

L. LABONTE, LPMC

D. SAVIN, University of Brunel (UK) 


\section{Statistics of resonance states in a weakly open chaotic microwave cavity}

Université

nice sophia antipo
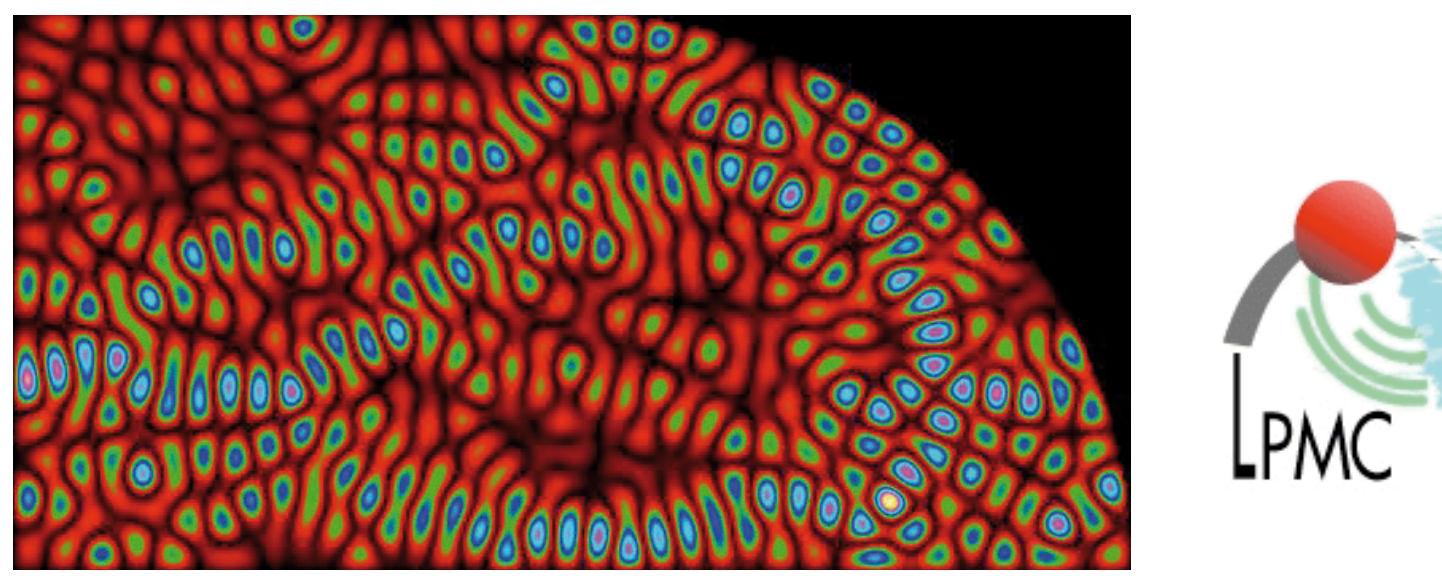

C. POLI, O. LEGRAND and F. MORTESSAGNE

Laboratoire de Physique de la Matière Condensée (LPMC) CNRS UMR 6622 \& Université de Nice-Sophia Antipolis

in collaboration with

D. SAVIN, University of Brunel (UK)

L. LABONTE, LPMC 


\section{Various experimental open systems}

Optical microcavities

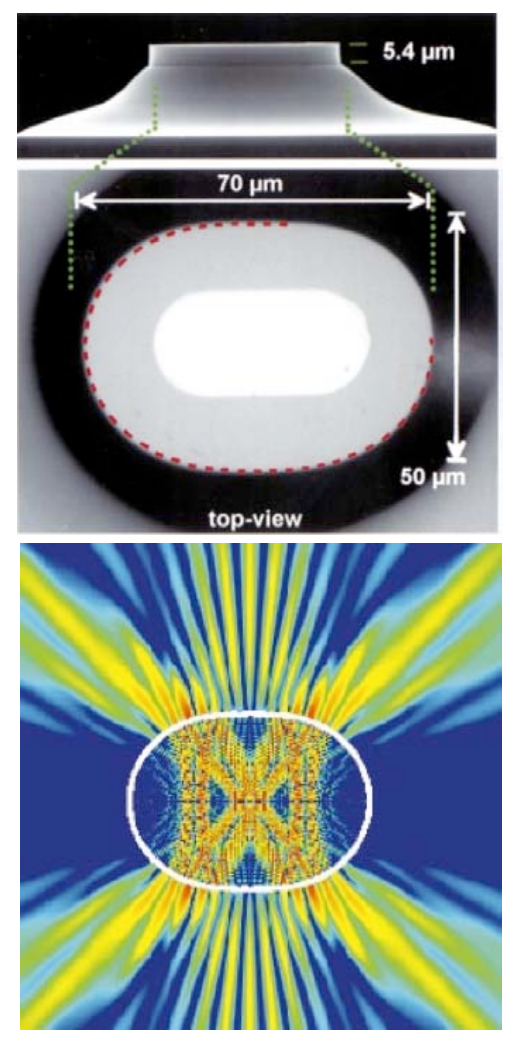

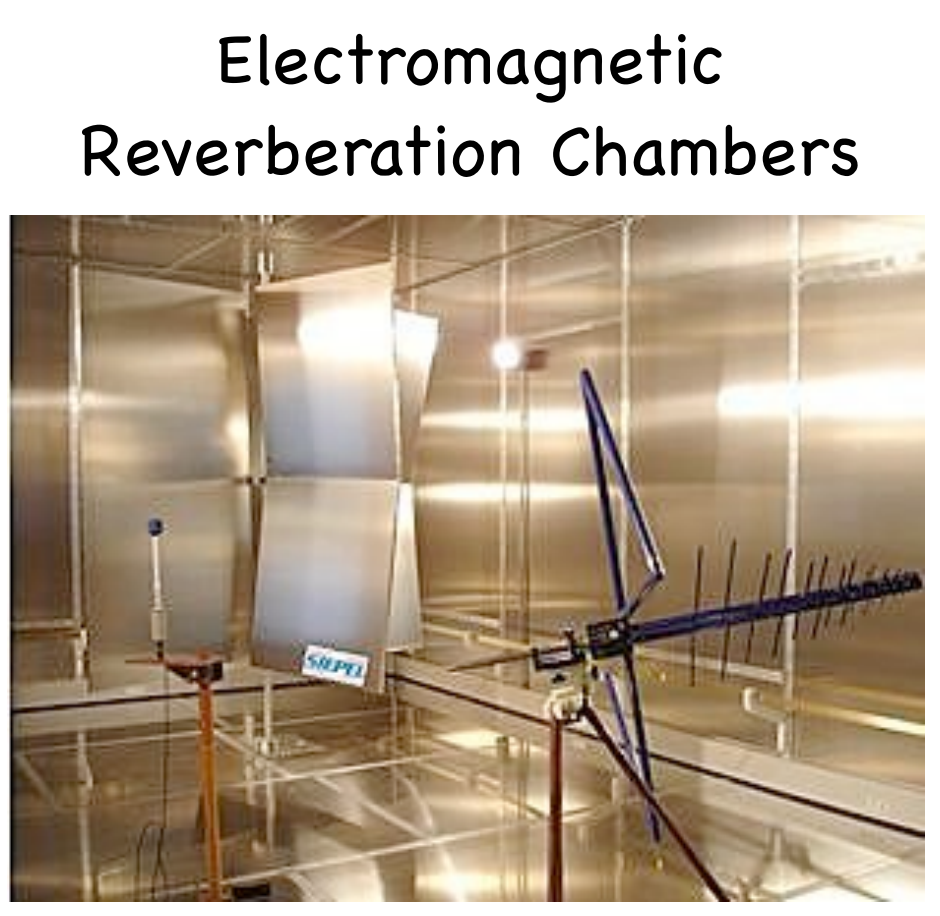

Electromagnetic

Reverberation Chambers

Coupling to the environment has to be taken into account to understand realistic systems. 


\section{Modeling open systems}

\section{Effective Hamiltonian formalism}

Losses are modelled by fictitious open channels

$V_{n j}$ couples the nth resonance to the jth channel

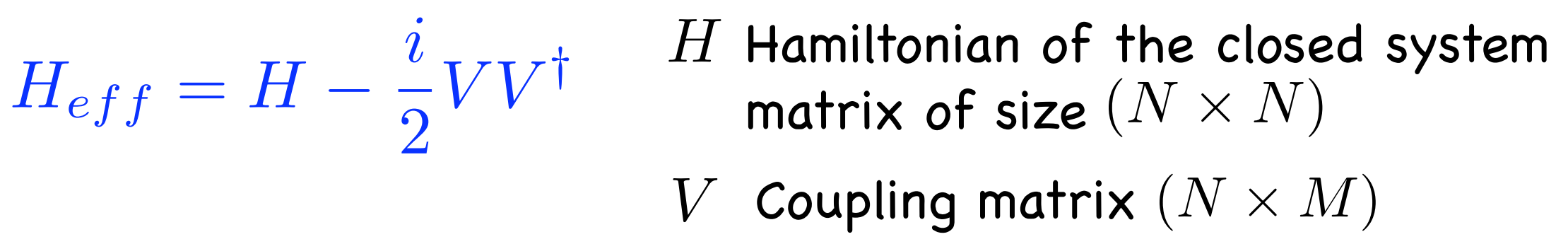

Diagonalization:

$$
\mathcal{H}_{\text {eff }}\left|\psi_{n}\right\rangle=\left(E_{n}-(i / 2) \Gamma_{n}\right)\left|\psi_{n}\right\rangle
$$




\section{Outline}

Statistics of open chaotic wave systems

Statistics of spectral widths (a brief reminder)

Properties of the complex field

Petermann factor, Phase rigidity and Complexness parameter

Description of the statistical models

The 2-level model

The $\mathrm{N}$-level model

Statistics of the complexness parameter for the $\mathrm{N}$-level

Average Complexness parameter and widths fluctuations

Distribution function for $G O E$

Statistics of a 2D chaotic open microwave cavity

FEM numerical results with Ohmic losses at the boundary

Distribution of the complexness parameter 


\section{Statistics of the spectral widths}

Statistics of the spectral widths are well-known from the regime of isolated resonances to the overlapping regime.

Regime of isolated resonances $\langle\Gamma\rangle \ll \Delta$

Using Gaussian coupling amplitudes: The spectral widths are $\chi^{2}$ distributed

Regime of overlapping resonances $\langle\Gamma\rangle \succeq \Delta$

Theoretical study

H. -J. Sommers, Y. Fyodorov and M.Titov, J. Phys. A. 32, L77 (I 999)

Experimental study

U. Kuhl, R. Höhmann, J. Main and H.-J. Stöckmann, Phys. Rev. Lett. I 00, 254 I 0 I (2008)
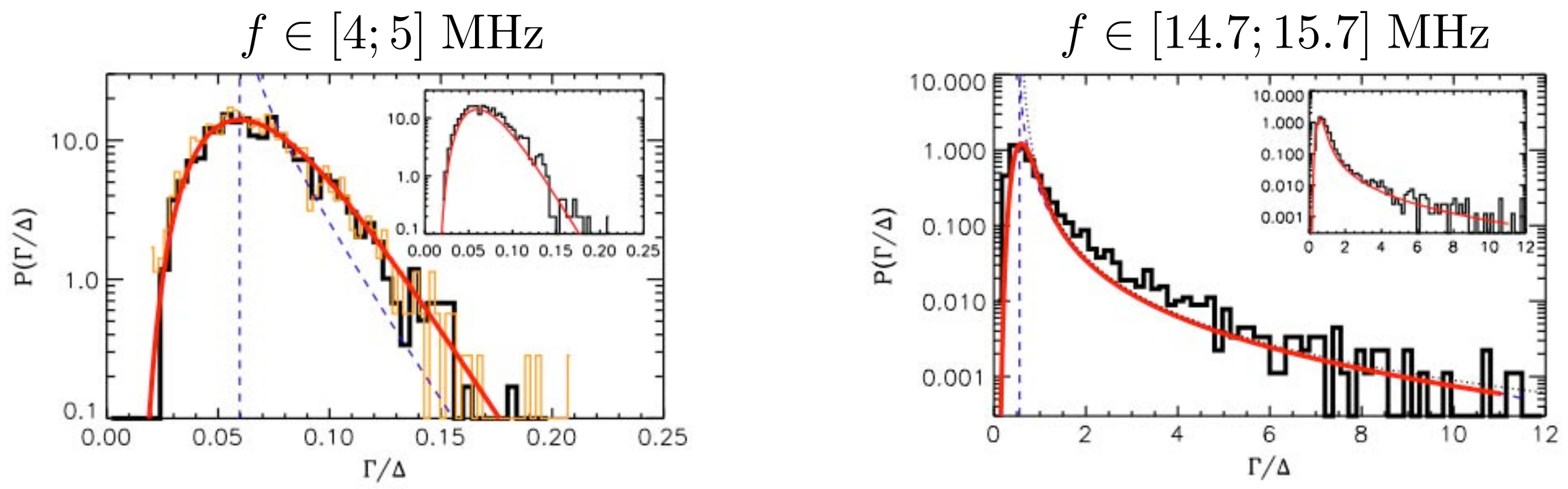


\title{
Properties of the eigenfunctions CLOSED CHAOTIC SYSTEM with TRS
}

The system is described by a real Hermitian Hamiltonian

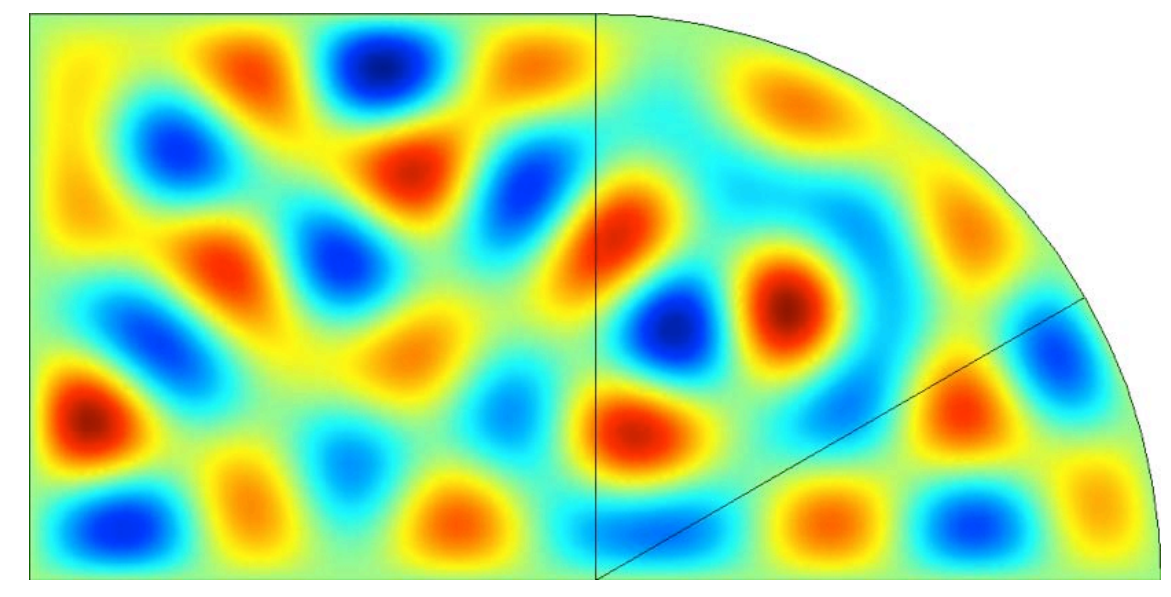

\author{
eigenfunctions are real
} and orthogonal:

$$
\int \psi_{n} \psi_{m}=\delta_{n m}
$$

The field may be viewed as a random

Gaussian variable (M.V. Berry J. Phys A: Math. Gen. I 0, 2083 (1977) )

Direct observation of Berry's hypothesis in a chaotic optical fiber:

Doya et al. PRE 65, 056223 (2002). 


\section{Properties of the eigenfunctions OPEN CHAOTIC SYSTEM}

The system is described by a non Hermitian Hamiltonian

The eigenfunctions are complex: $\psi_{n}=\psi_{n}^{R}+i \psi_{n}^{I}$ and bi-orthogonal:

$\int\left[\psi_{n}^{R} \psi_{m}^{R}-\psi_{n}^{I} \psi_{m}^{I}\right]=\delta_{n m} \quad \int\left[\psi_{n}^{R} \psi_{m}^{I}+\psi_{n}^{I} \psi_{m}^{R}\right]=0$

Real part of the field

Imaginary part of the field
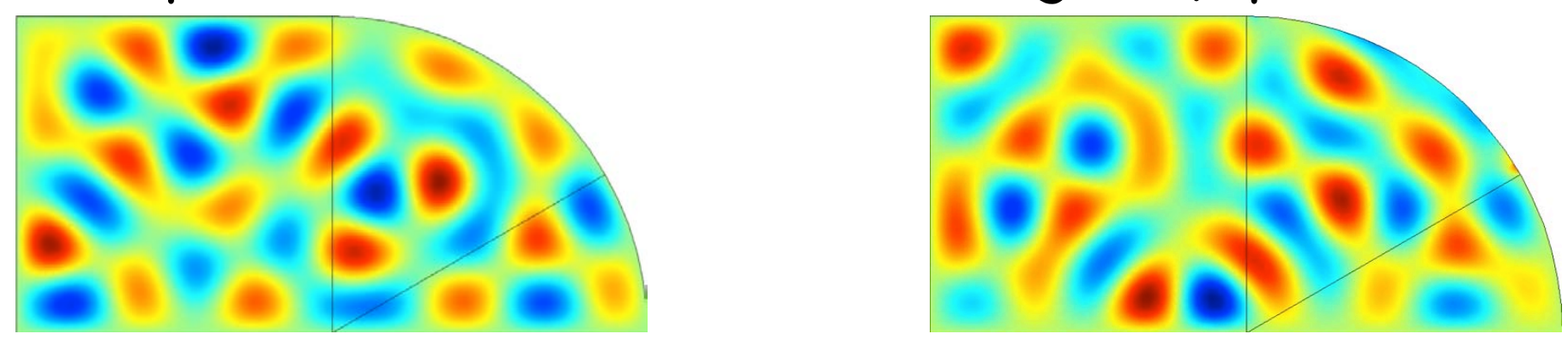

$\psi_{n}^{R} \quad \psi_{n}^{I}$ may be viewed as independent random

Gaussian variables (R. Pnini and B. Shapiro, Phys. Rev. E, 54 (1996) RI032.) 


\section{Properties of the complex field}

The Petermann Factor $K$

K. Petermann, I.E.E.E. QE- I 5, 566 (1979)

A. E. Siegman, Phys. Rev.A 39, 1253 (1989)

$$
K=\left|\frac{\int d \vec{r}\left[\psi_{R}^{2}+\psi_{I}^{2}\right]}{\int d \vec{r}\left[\psi_{R}^{2}-\psi_{I}^{2}\right]}\right|^{2}
$$

\section{LINE WIDTH OF A LASER}

Schalow-Townes line width

$\delta \omega_{S T}=\hbar \omega \frac{\Gamma^{2}}{2 P_{\text {out }}} \quad \begin{aligned} & \Gamma \text { spectral width of the passive cavity } \\ & P_{\text {out }} \text { output power }\end{aligned}$

True fundamental limit of line width

$\delta \omega \propto K \delta \omega_{S T}$

The enhancement factor called the Petermann factor characterizes the non orthogonality of the resonance modes 


\section{Properties of the complex field}

The phase rigidity $\rho^{2}$

P. Brouwer, P.R.E 68, 246205 (2003)

Statistics of wavefunctions in

$$
\rho=\frac{\int d \vec{r}\left[\psi_{R}^{2}-\psi_{I}^{2}\right]}{\int d \vec{r}\left[\psi_{R}^{2}+\psi_{I}^{2}\right]}
$$

open chaotic billiard

\section{Y.-H Kim .et al., P.R.L. 94, 036804, (2005)}

Measurement of Long-Range Wave-Function Correlations in an Open Microwave Billiard
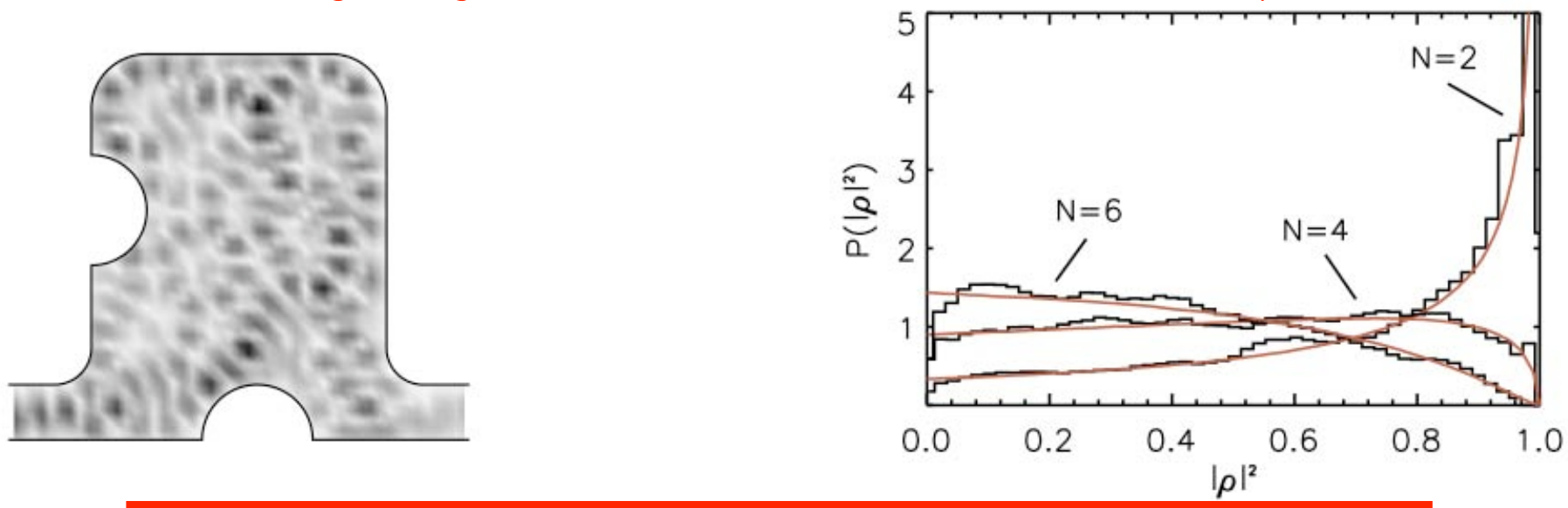

The phase rigidity was generally studied as a continuous function of the frequency 


\section{Properties of the complex field}

The complexness parameter:

O. Lobkis and R. Weaver, JASA I 08, I 480 (2000) Complex modal statistics in a reverberant dissipative body

$$
q_{n}^{2}=\frac{\int d \vec{r}\left(\psi_{n}^{I}\right)^{2}}{\int d \vec{r}\left(\psi_{n}^{R}\right)^{2}}
$$

J. Barthélemy et. al., Euro. Phys. Lett. 70, 162 (2005)

Inhomogeneous resonance broadening and statistics of complex functions in a chaotic microwave cavity

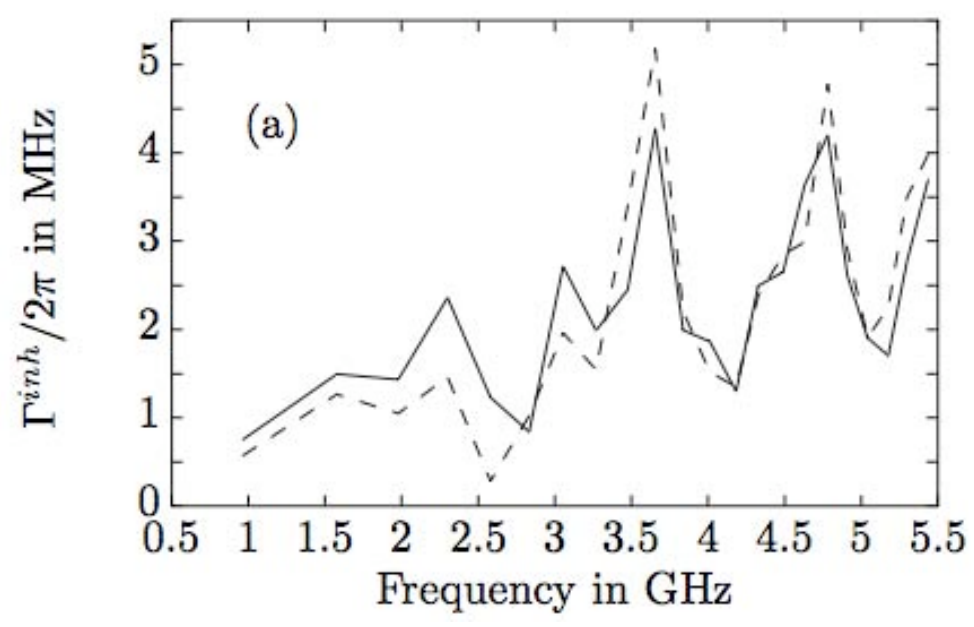

Through a statistical ray model:

$$
q_{n}=\frac{\pi S}{c P} \Gamma_{n}
$$




\section{Relations between Phase rigidity, Petermann factor and Complexness parameter}

For a given resonance state, the quantities are closely related

Using the complexness parameter: $q_{n}^{2}=\frac{\int d \vec{r}\left(\psi_{n}^{I}\right)^{2}}{\int d \vec{r}\left(\psi_{n}^{R}\right)^{2}}$

$$
K_{n}=\left(\frac{1+q_{n}^{2}}{1-q_{n}^{2}}\right)^{2}
$$$$
\rho_{n}^{2}=\left(\frac{1-q_{n}^{2}}{1+q_{n}^{2}}\right)^{2}
$$

The eigenfunction statistics are investigated via the complexness parameter 
Statistics of open chaotic wave systems

Statistics of spectral widths (a brief reminder)

Properties of the complex field

Petermann factor, Phase rigidity and Complexness parameter

Description of the statistical models

The 2-level model

The $\mathrm{N}$-level model

Statistics of the complexness parameter for the $\mathrm{N}$-level

Average Complexness parameter and widths fluctuations

Distribution function for GOE

Statistics of a 2D chaotic open microwave cavity

FEM numerical results with Ohmic losses at the boundary

Distribution of the complexness parameter 


\section{The 2-level model}

D. Savin, et. al. Europhys. Lett. 76, 774 (2006)

Inhomogeneous losses and complexness of wave functions in chaotic cavities

Hypothesis: The complexness parameter is a local quantity

$H_{e f f}=\left(\begin{array}{cc}E_{1} & 0 \\ 0 & E_{2}\end{array}\right)-\frac{i}{2}\left(\begin{array}{ll}\Gamma_{11} & \Gamma_{12} \\ \Gamma_{21} & \Gamma_{22}\end{array}\right)$ with $\Gamma_{n p}=\sum_{c=1}^{M} V_{n}^{c} V_{p}^{c}$

In the weak coupling regime: $\langle\Gamma\rangle=M \sigma^{2} \ll \Delta$

where $\left\langle\left(V_{n}^{c}\right)^{2}\right\rangle=\sigma^{2}$

The complexness parameter of both resonances reads:

$$
q^{2}=\frac{\Gamma_{12}^{2}}{4\left(E_{2}-E_{1}\right)^{2}}
$$




\section{Complexness of elastic modes of a chaotic silicon wafer}

O. Xeridat, C. Poli, O. Legrand, F. Mortessagne, and P. Sebbah, PRE 80 (2009)
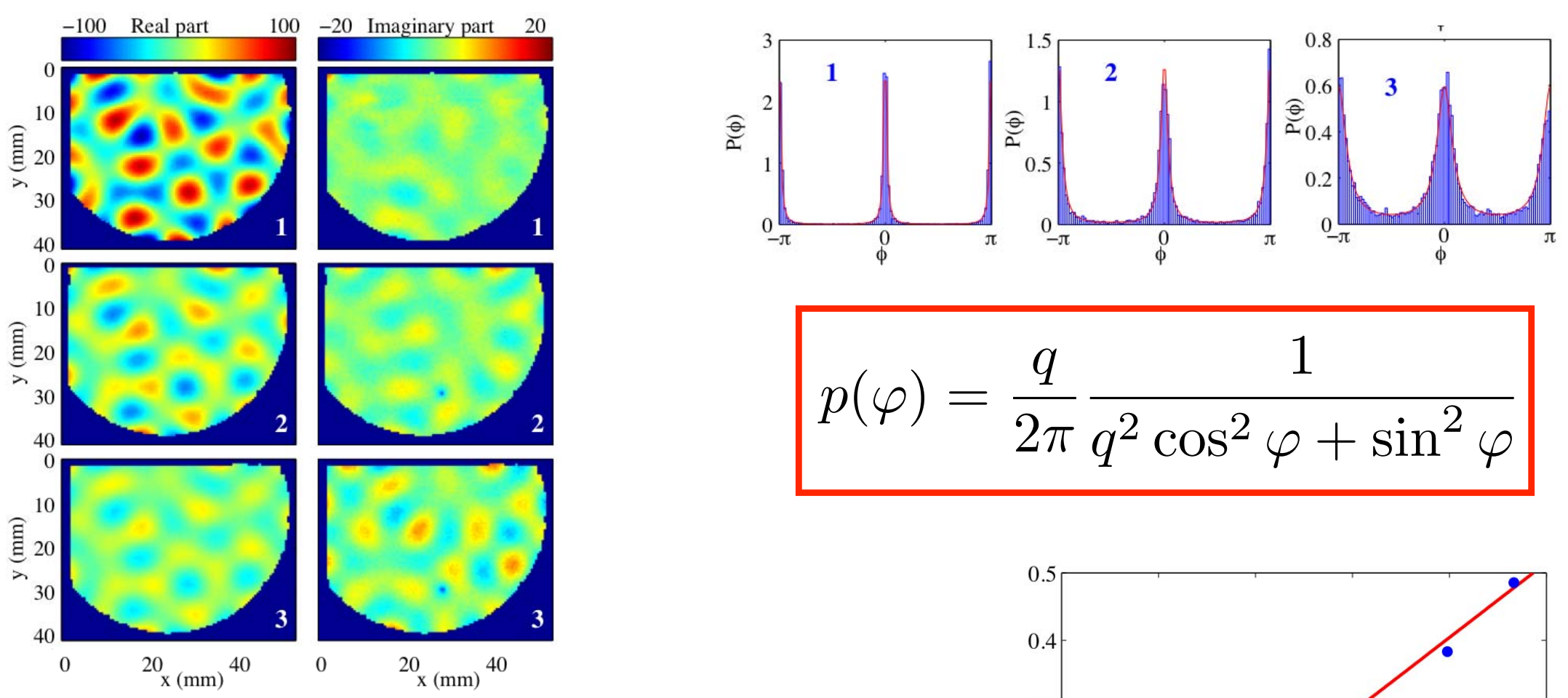

$$
p(\varphi)=\frac{q}{2 \pi} \frac{1}{q^{2} \cos ^{2} \varphi+\sin ^{2} \varphi}
$$

$$
q_{n}=\frac{\sqrt{\left(\sum_{c} V_{1}^{c} V_{2}^{c}\right)^{2}}}{\sum_{c}\left(V_{n}^{c}\right)^{2}} \frac{\Gamma_{n}}{2\left(E_{2}-E_{1}\right)}
$$

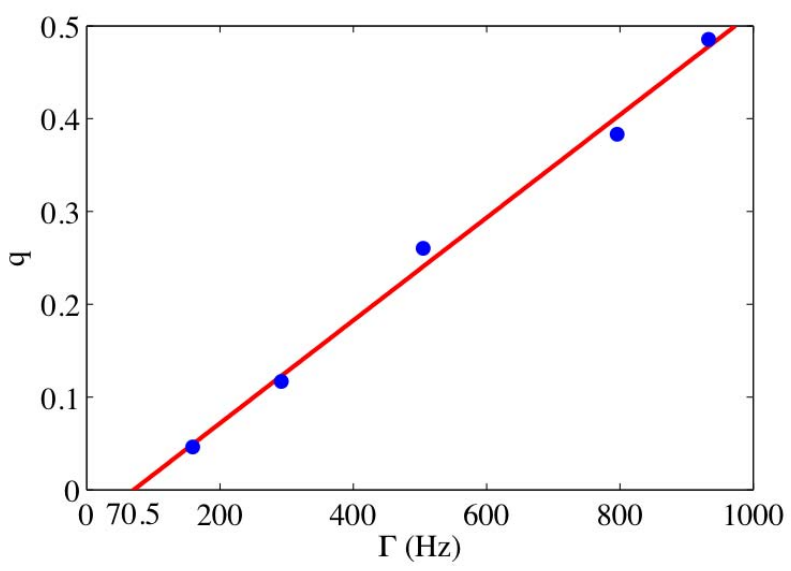




\section{The $\mathrm{N}$-level model}

Effective Hamiltonian in the eigenbasis of $\mathrm{H}$ :

$$
H_{\text {eff }}=\left(\begin{array}{ccc}
E_{1} & 0 & 0 \\
0 & \ddots & 0 \\
0 & 0 & E_{N}
\end{array}\right)-\frac{i}{2}\left(\begin{array}{ccc}
\Gamma_{11} & \cdots & \Gamma_{1 N} \\
\vdots & \ddots & \vdots \\
\Gamma_{N 1} & \cdots & \Gamma_{N N}
\end{array}\right) \Gamma_{n p}=\sum_{c=1}^{M} V_{n}^{c} V_{p}^{c}
$$

Non-overlapping regime

Eigenvalues:

$$
\mathcal{E}_{n}=E_{n}-\frac{i}{2} \Gamma_{n} \quad \text { with } \quad \Gamma_{n}=\sum_{c=1}^{M}\left(V_{n}^{c}\right)^{2}
$$

$\left\{E_{n}\right\}$ statistics correspond to the closed case

$\left\{\Gamma_{n}\right\}$ are $\chi^{2}$ distributed 


\section{The $\mathrm{N}$-level model (2)}

Eigenvectors: $\quad\left|\psi_{n}\right\rangle=|n\rangle-i \sum_{p \neq n} \frac{\Gamma_{n p}}{2\left(E_{n}-E_{p}\right)}|p\rangle$

with $\Gamma_{n p}=\sum_{c=1}^{M} V_{n}^{c} V_{p}^{c} \rightarrow \begin{gathered}\text { interference of resonance states } \\ \text { due to common decay channels }\end{gathered}$

where $\{|n\rangle\}$ is the eigenbasis of the closed part

Expression of the complexness parameter:

$$
q_{n}^{2}=\sum_{p \neq n} \frac{\Gamma_{n p}^{2}}{4\left(E_{n}-E_{p}\right)^{2}}
$$

Sum of correlated random variables 


\section{Statistical ensembles of $\mathrm{H}$ : \\ The Gaussian Orthogonal Ensemble}

Statistics of chaotic closed systems with TRS are described by GOE:

$$
p(H) \propto \exp \left(-\frac{N \pi^{2}}{4} \operatorname{Tr} H^{2}\right)
$$

EIGENENERGIES

$$
\begin{gathered}
p\left(E_{1}, \ldots, E_{N}\right) \propto \prod_{n>m}\left|E_{n}-E_{m}\right| \exp \left(-\frac{N \pi^{2}}{4} \sum_{n} E_{n}^{2}\right) \\
\text { linear level repulsion }
\end{gathered}
$$

The spectrum of $H$ exhibits fluctuations and rigidity

EIGENVECTORS

The $\left\{\psi_{n}\right\}$ are Gaussian distributed 
Statistics of open chaotic wave systems

Statistics of spectral widths (a brief reminder)

Properties of the complex field

Petermann factor, Phase rigidity and Complexness parameter

Description of the statistical models

The 2-level model

The $\mathrm{N}$-level model

Statistics of the complexness parameter for the $\mathrm{N}$-level

Average Complexness parameter and widths fluctuations

Distribution function for GOE

Statistics of a 2D chaotic open microwave cavity

FEM numerical results with Ohmic losses at the boundary

Distribution of the complexness parameter 


\section{Average Complexness parameter and widths fluctuations (1)}

Rescaled parameters and their statistics

Dimensionless spectral width $\gamma_{n}$

$\gamma_{n}=\frac{\Gamma_{n}}{\sigma^{2}}$

$\chi^{2}$-distributed :

$\langle\gamma\rangle=M \quad$ and $\quad \operatorname{var}(\gamma)=2 M=\frac{2}{M}\langle\gamma\rangle^{2}$

Distribution: $P_{M}(\gamma)=\frac{1}{2^{M / 2} \Gamma(M / 2)} \gamma^{M / 2-1} e^{-\gamma / 2}$

Rescaled complexness parameter $X_{n}$

$0 \leq q_{n}^{2} \leq 1 \quad$ then $\quad 0 \leq X_{n} \leq \frac{\Delta^{2}}{\sigma^{4}}$

$X_{n}=\frac{\Delta^{2}}{\sigma^{4}} q_{n}^{2}$

Weak coupling regime: $\frac{\Delta^{2}}{\sigma^{4}} \gg 1$ 


\section{Sokolov's approach}

V.V. Sokolov, et.V.G. Zelevinsky Nucl. Phys. A504, 562 (I 989)

Dynamics and statistics of unstable quantum states

$$
\Gamma_{n p}=\sum_{c=1}^{M} V_{n}^{c} V_{p}^{c}
$$

viewed as a scalar product of $M$-dimensional vectors

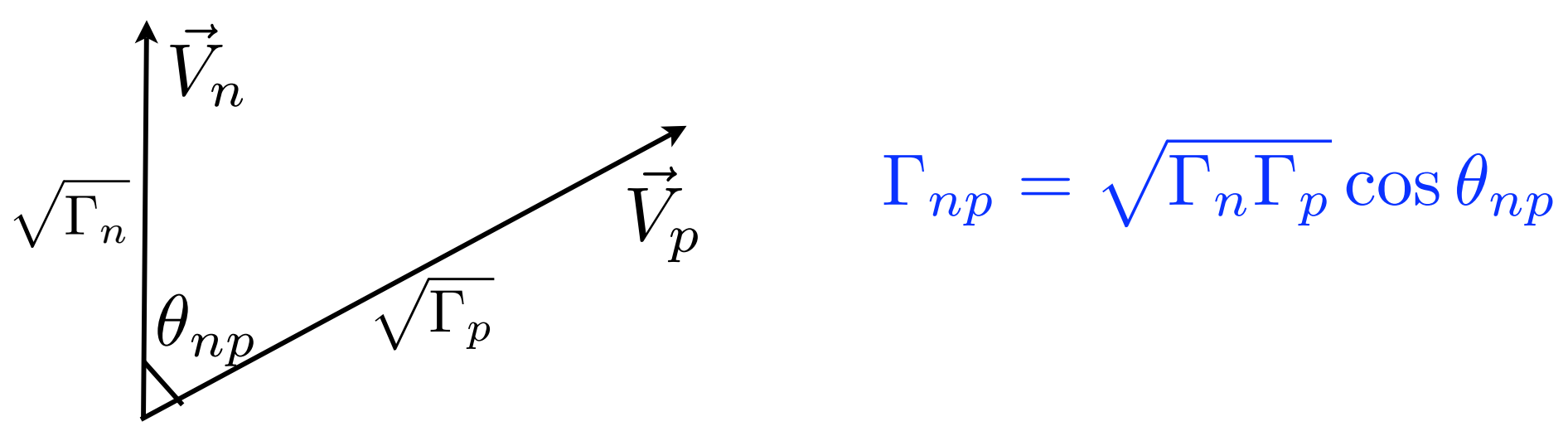

The angle is distributed for $M>1$ as:

$$
p_{M}(\theta)=\frac{\Gamma(M / 2)}{\sqrt{\pi} \Gamma((M-1) / 2)} \sin ^{M-2} \theta
$$


Average Complexness parameter and width fluctuations

(2)

$q_{n}^{2}=\sum_{p \neq n} \frac{\Gamma_{n p}^{2}}{4\left(E_{n}-E_{p}\right)^{2}}$ yields $X_{n}=\gamma_{n} \sum_{p \neq n} \frac{\Delta^{2} Z_{p}}{4\left(E_{n}-E_{p}\right)^{2}}$

where: $Z_{p}=\gamma_{p} \cos ^{2} \theta_{n p}$

Integrating over $\gamma$ and $\theta$ in the definition $P(Z)=\left\langle\delta\left(Z-\gamma \cos ^{2} \theta\right)\right\rangle$ yields:

$$
P(Z)=\frac{1}{\sqrt{2 \pi Z}} e^{-Z / 2}
$$

independent of $M$

Since $\langle\gamma\rangle=M$ and $\langle Z\rangle=1=\langle\gamma\rangle\left\langle\cos ^{2} \theta\right\rangle$

$$
\begin{array}{ll}
\text { one obtains: } & \langle X\rangle=M f \text { where } f=\left\langle\sum_{p \neq n} \frac{\Delta^{2}}{4\left(E_{n}-E_{p}\right)^{2}}\right\rangle \\
\text { and also: } & \langle X\rangle=\frac{f}{2} \operatorname{var}(\gamma)=\frac{f}{M}\langle\gamma\rangle^{2}
\end{array}
$$




\section{Distribution function of $X$ for $G O E$}

$$
\left\langle q^{2}\right\rangle=M f \frac{\sigma^{4}}{\Delta^{2}}
$$$$
\text { with } f=\left\langle\sum_{p \neq n} \frac{\Delta^{2}}{4\left(E_{n}-E_{p}\right)^{2}}\right\rangle_{n}
$$

considering the continuous limit: $\quad f=\frac{1}{2} \int_{0}^{\infty} d x x^{-2} R_{2}(x)$

2-point correlation function at small distance: $R_{2}(x) \sim x$

The average of the complexness factor has

a logarithmic divergence

Summation of the perturbation series is required But... for all practical purposes, regularized by:

$$
f=\frac{1}{2} \int_{\bigodot}^{\infty} d x x^{-2} R_{2}(x)
$$




\section{Probability distribution of the} complexness parameter for GOE

$$
\mathcal{P}_{M}^{\text {goe }}(X)=\frac{\pi^{2} M}{24 X^{2}} \frac{1+\frac{\pi^{2}(3+M)}{4 X}}{\left(1+\frac{\pi^{2}}{4 X}\right)^{M / 2+2}}
$$

C. Poli, D. Savin, O. Legrand and F. Mortessagne PRE 80, 046203 (2009)

$$
M=1
$$

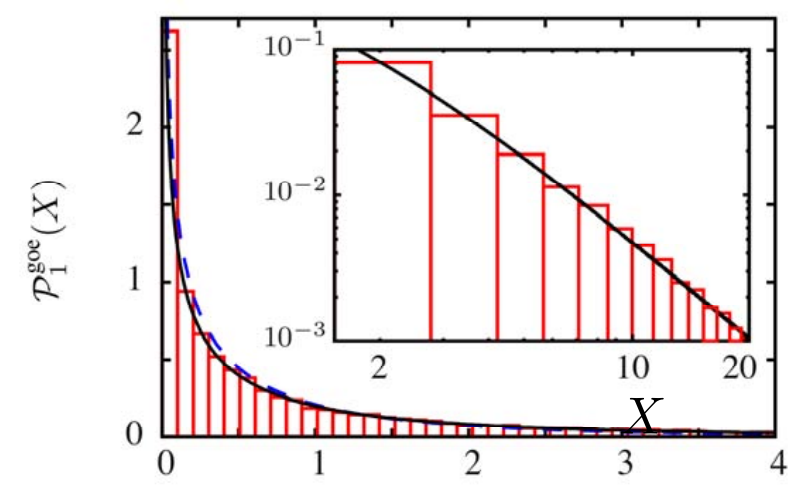

$M=5$

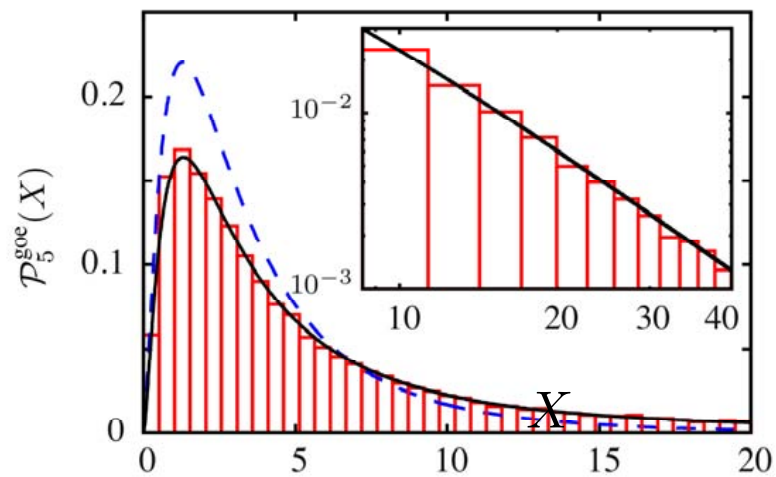

$M=10$

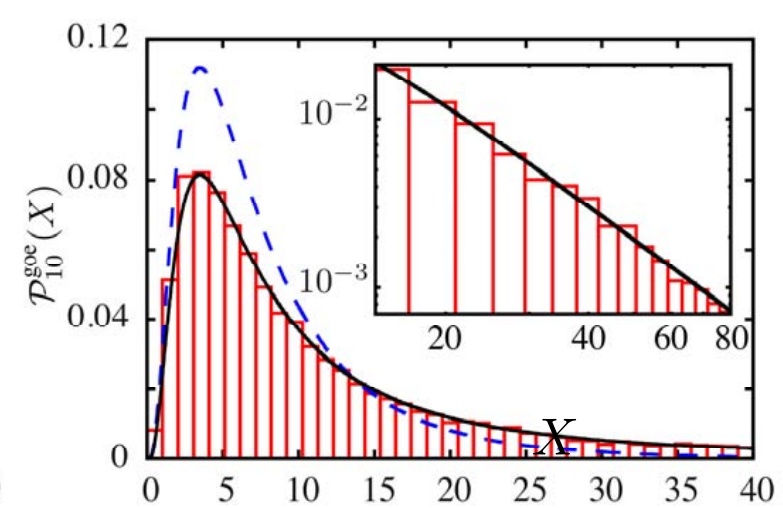


Statistics of open chaotic wave systems

Statistics of spectral widths (a brief reminder)

Properties of the complex field

Petermann factor, Phase rigidity and Complexness parameter

Description of the statistical models

The 2-level model

The $\mathrm{N}$-level model

Statistics of the complexness parameter for the $\mathrm{N}$-level

Average Complexness parameter and widths fluctuations

Distribution function for GOE

Statistics of a 2D chaotic open microwave cavity

FEM numerical results with Ohmic losses at the boundary

Distribution of the complexness parameter 


\section{D open Chaotic Microwave Cavity}

TM polarized electromagnetic field

$$
\psi(\mathbf{r})=E_{z}(\mathbf{r})
$$

solution of

$$
-\left(\frac{\partial^{2}}{\partial x^{2}}+\frac{\partial^{2}}{\partial y^{2}}\right) \psi(\mathbf{r})=\frac{\tilde{\omega}^{2}}{c^{2}} \psi(\mathbf{r}) \quad \begin{gathered}
\tilde{\omega} \\
\text { complex angular } \\
\text { frequency }
\end{gathered}
$$

subject to non-Hermitian B.C.S (Ohmic losses)

finite conductivity imposed on a length $l_{a b s}$ of the contour

sliding half-disk

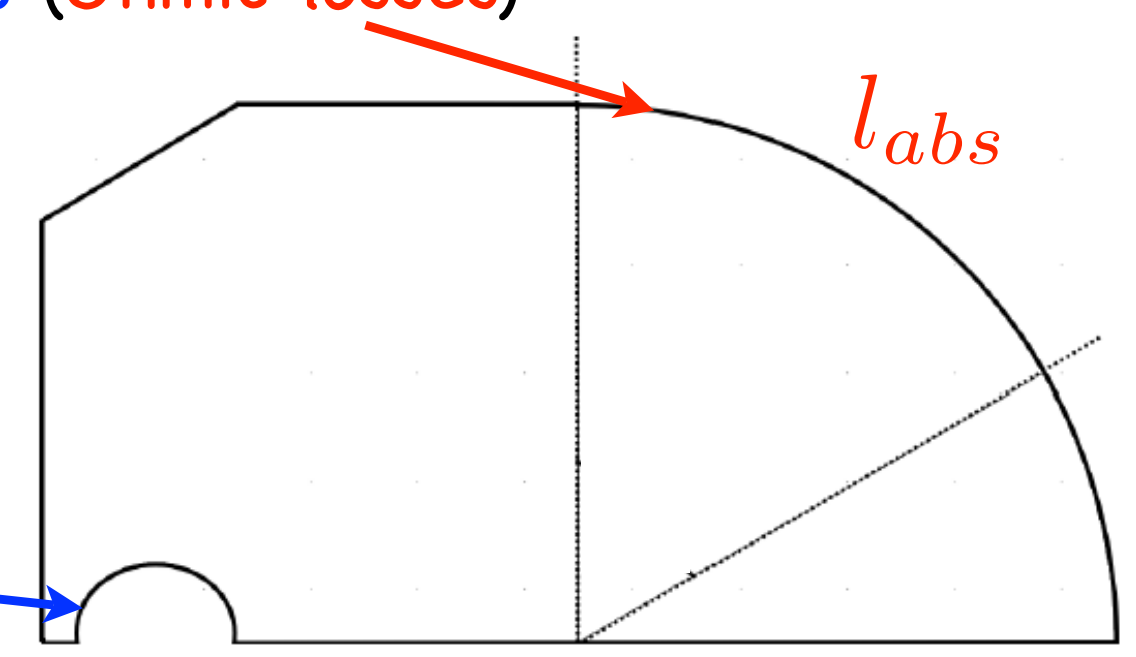




\section{Real (left) and Imaginary (right) components of the 500th resonance state}

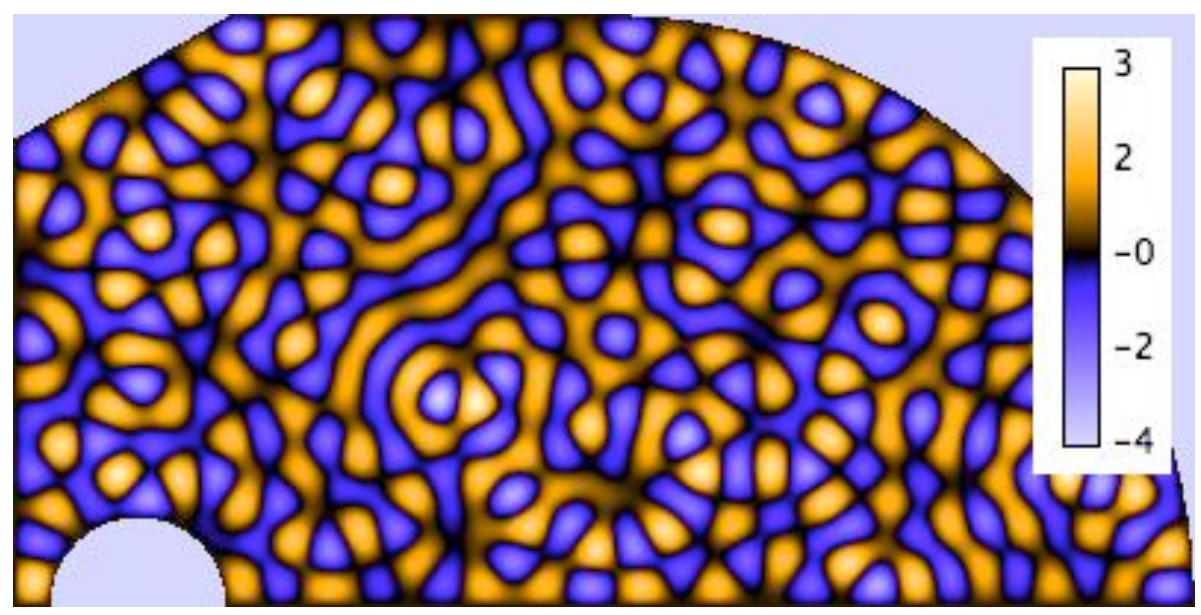

solved by FEM with Comsol ${ }^{\mathrm{TM}}$

Complex eigenvalues

$\tilde{\omega}_{n}=\omega_{n}-i \zeta_{n} / 2$

Usual correspondance

$\left(E_{n}, \Gamma_{n}\right)$ with $\left(\omega_{n}^{2}, \omega_{n} \zeta_{n}\right)$






\section{Numerical results}

- Intervals of 100 consecutive resonances above the 300th

- 21 different positions of the movable half-disk

The number of channels : $M=\frac{l_{a b s}}{\lambda / 2}$

To explore various numbers of channels,

2 frequency ranges:

- from the 300th to the 400th resonances $(\Delta M / M \simeq 14 \%)$

- from the 700th to the 800th resonances $(\Delta M / M \simeq 7 \%)$

3 different lengths :

$l_{a b s}=\pi / 18, \pi / 6, \pi / 2$

Weak coupling regime : $\sqrt{\operatorname{var}(\Gamma)} / \Delta<10^{-2}$ 
(a) resonances 300 to 400 .

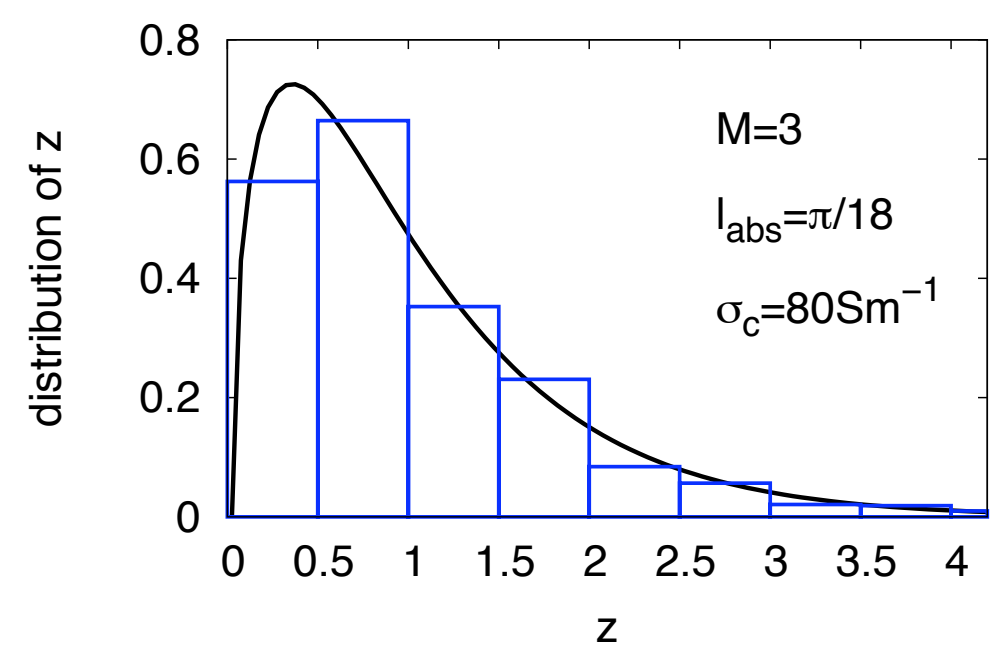

(c) resonances 700 to 800 .

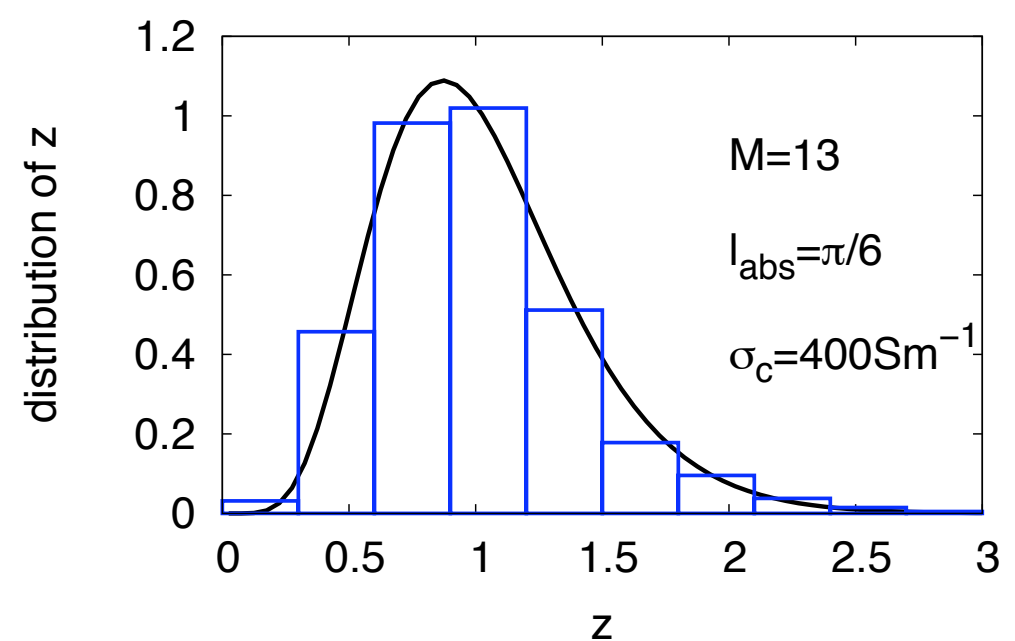

Distribution of $\mathrm{z}=\gamma /\langle\gamma\rangle$ $M=\frac{l_{a b s}}{\lambda / 2} \simeq \frac{2}{\left\langle\gamma^{2}\right\rangle /\langle\gamma\rangle^{2}-1}$ (b) resonances 300 to 400 .

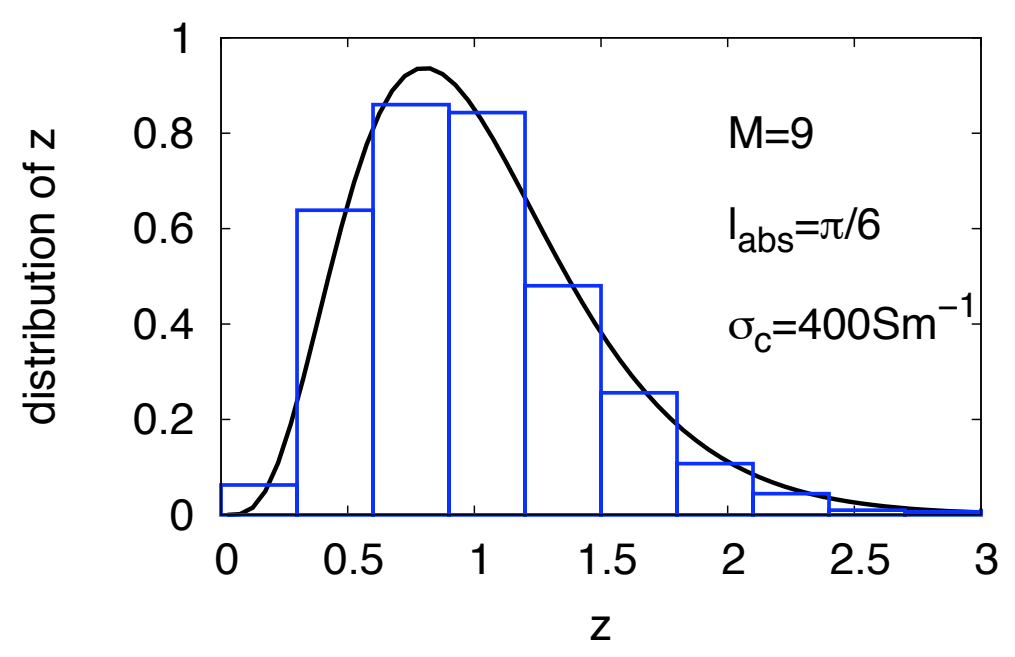

(d) resonances 700 to 800 .

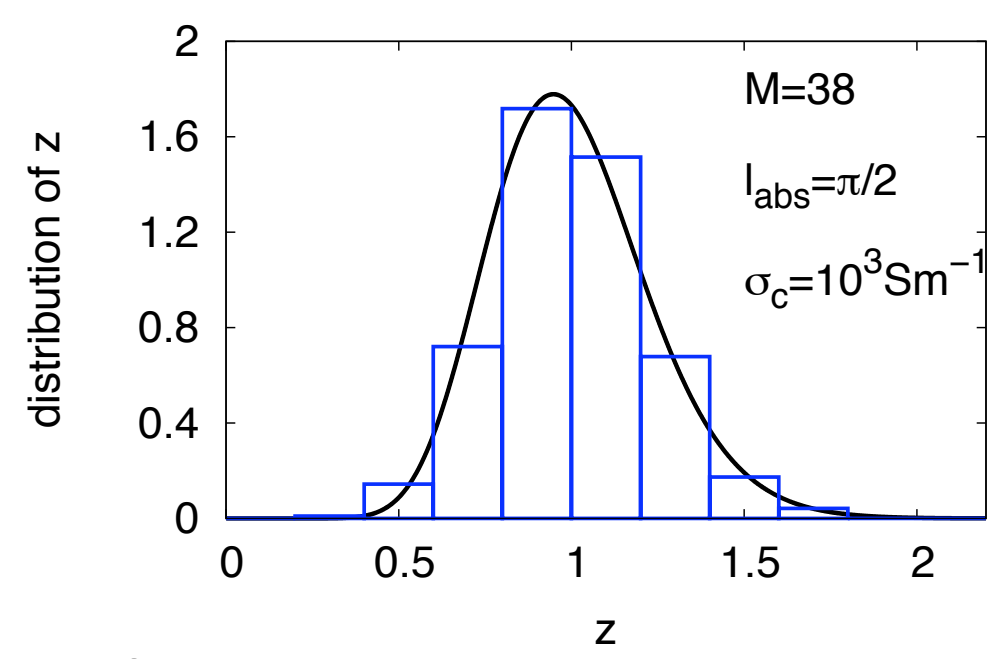

$\chi^{2}$-distribution : solid line No adjustable parameter! 


\section{Numerical distribution of the complex parameter}

(a) resonances 300 to 400 .

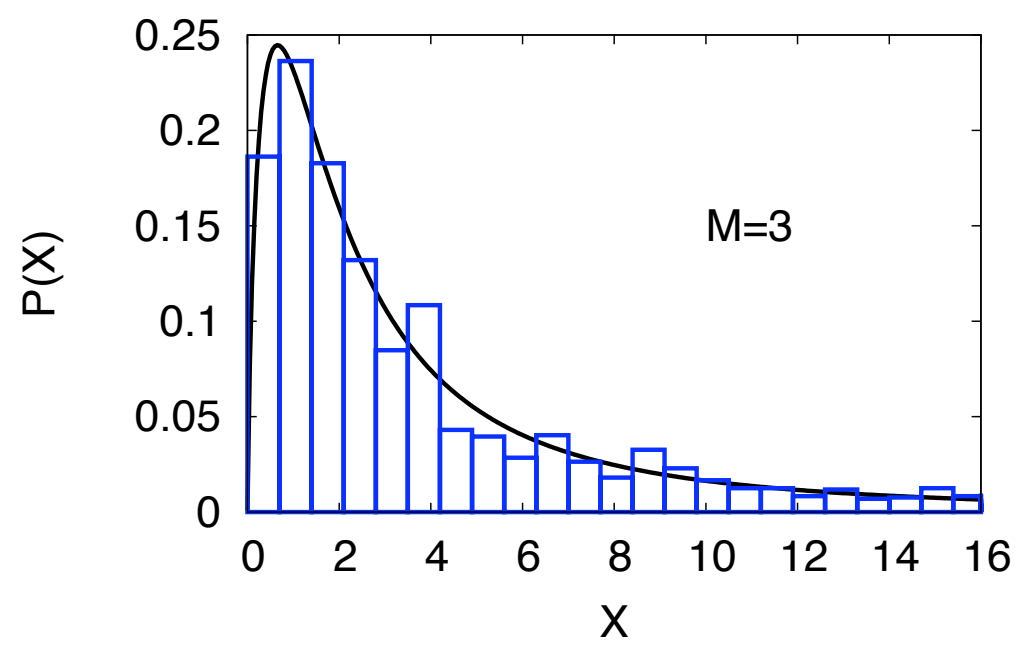

(c) resonances 700 to 800 .

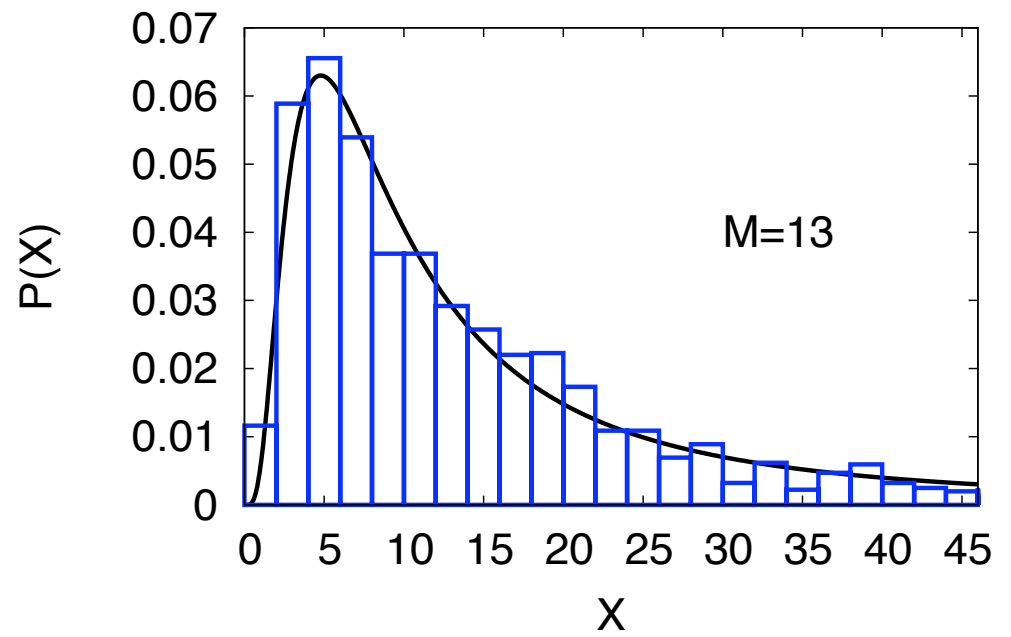

(b) resonances 300 to 400

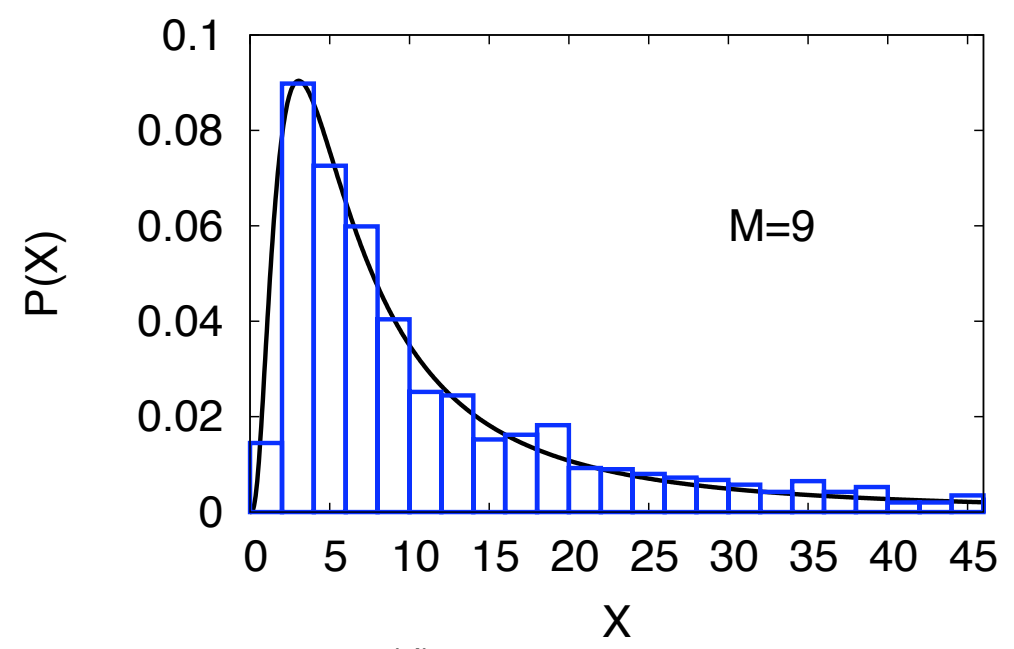

(d) resonances 700 to 800

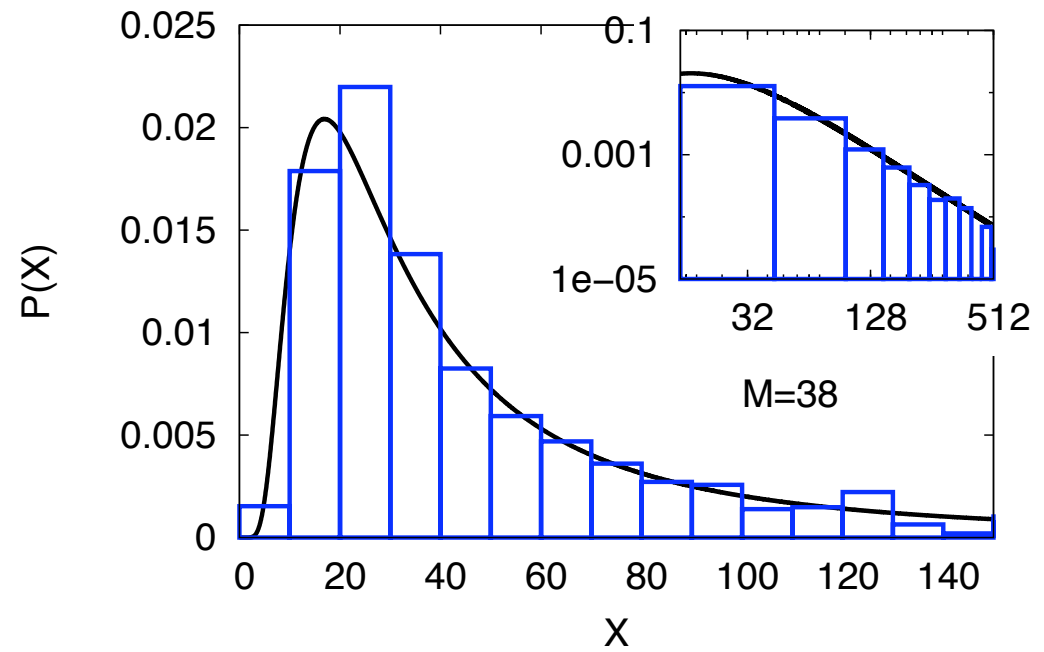

$\mathcal{P}_{M}^{G O E}(X)$ : solid line 


\section{Summary}

Complexness of eigenfunctions was studied using the effective Hamiltonian formalism \& RMT

Proportionality between the average complexness parameter and the variance of the resonance width

Exact probability distribution of the complexness parameter derived in the GOE case

Spatially continuously distributed losses in a chaotic cavity described by a Random Matrix model with a finite number $M$ of coupling channels, which constitutes a variable parameter in the cavity. 